\title{
SYNTHESIS AND CHARACTERIZATION OF NEW AMINO ACYL-4-THIAZOLIDONES
}

\author{
Ana Cristina Lima Leite*, Luciene Maria F. Santos, Diogo Rodrigo de M. Moreira and Dalci José Brondani \\ Departamento de Ciências Farmacêuticas, Universidade Federal de Pernambuco, Rua Prof. Artur Sá, s/n, \\ 50740-520 Recife - PE, Brasil
}

Recebido em 6/9/05; aceito em 4/5/06; publicado na web em 26/9/06

\begin{abstract}
A series of heterocyclic compounds with a 4-thiazolidone nucleus and amino acyl moiety were synthesized by protection reaction of thiosemicarbazide using the symmetrical anhydride (Boc) $)_{2} \mathrm{O}$ and cyclization with chloroacetic acid under mild conditions. Trifluoroacetic acid was used to obtain 4-thiazolidone and the $\alpha$-amino acid condensation reactions were carried out using strategies for peptide synthesis. The characterization of this new class of compounds was performed using IR and ${ }^{1} \mathrm{H}-\mathrm{NMR}$ spectroscopy.
\end{abstract}

Keywords: 4-thiazolidone; peptidyl derivatives; heterocyclic compounds.

\section{INTRODUCTION}

A wide range of biological activities of thiazole containing compounds has been reported. Further reports regarding their synthesis, properties, reactions and applications have led to an increased interest in related thiazolidones ${ }^{1}$. Thiazole and thiazolidone nuclei exhibit a number of biological properties, such as antimicrobial $^{2-4}$, antiprotozoal ${ }^{5-6}$ antiinflammatory ${ }^{2,7}$, anticonvulsant ${ }^{8}$, cardiotonic $^{9}$, analgesic and antithermic ${ }^{10}$ and anticancer ${ }^{11}$ properties, among others.

A group of cyclic peptides containing thiazolic heterocyclic rings linked to amino acid blocks were isolated and characterised from nature. It was found that some of these marine cyclopeptides demonstrated anticancer and antimicrobial properties ${ }^{12-16}$. In vitro biological screening suggests that many of these small cyclopeptides have potential therapeutic effects and are generally among the more promising lead structures ${ }^{12}$. The presence of an unusual amino acid and heterocyclic building blocks has stimulated interest in new synthetic methodologies and strategies to obtain a target structure ${ }^{16}$.

In addition to these findings, there is currently a tendency to use an amino acid moiety or a peptidyl residue during the prodrug design process. The literature reports that bioactive compounds show enhanced activity when linked to amino acids ${ }^{17-24}$.

As part of a research program for prodrug design, we performed the synthesis of a new series of compounds, containing a 4thiazolidone heterocyclic ring and an amino acid moiety. In the present paper we report the synthesis of new series of compounds and characterization of their structure by spectroscopic methods.

\section{EXPERIMENTAL}

\section{General}

All melting points were determined using a Thomas Hoover apparatus and are uncorrected. The IR spectra were obtained on a FTIR spectrophotometer Bruker, model IFS66 using KBr pellets. ${ }^{1} \mathrm{H}$ NMR spectra were registered on a Varian UNITYplus-300 MHz NMR spectrophotometer operating at a frequency of $300 \mathrm{MHz}$ for ${ }^{1} \mathrm{H}$ and $75 \mathrm{MHz}$ for ${ }^{13} \mathrm{C}$, using DMSO- $d_{6}$ as solvent and tetramethylsilane as the internal reference. The specific rotation of

*e-mail: acllb2003@yahoo.com.br compounds were obtained on a Perkin-Elmer, model 241 polarimeter. Thin-layer chromatography (TLC) was carried out on silica gel plates with fluorescence indicator $\mathrm{F}_{254}(0.2 \mathrm{~mm}$, E. Merck); the spots were visualised in UV light, and sprayed with a $2 \%$ ethanol solution of ninhydrin or charring reagent. Column chromatography was performed on silica using a Kiesegel 60 (230-400 Mesh, E. Merck), solvent system: ethyl acetate/ $n$-hexane (5/5). All reagents used in this study were of analytical grade.

1,N-[tert-butyloxycarbonyl-2,N-(aminomethanetione) hydrazide (2): A solution of thiosemicarbazide (1.0 g, $10 \mathrm{mmol}$ ), dioxane $(20 \mathrm{~mL})$, water $(10 \mathrm{~mL})$ and $1 \mathrm{~N} \mathrm{NaOH}(11 \mathrm{~mL})$ was stirred in an ice-water bath. Di-tert-butyl pyrocarbonate $(2.4 \mathrm{~g}, 11 \mathrm{mmol})$ was added and stirring was continued at room temperature for 30 min. The solution was concentrated in vacuo to about $15 \mathrm{~mL}$, cooled in an ice-water bath. Ethyl acetate $(30 \mathrm{~mL})$ was added and the solution acidified with dilute solution of $\mathrm{KHSO}_{4}$ to $\mathrm{pH}$ 2-3 (Congo paper). The aqueous phase was extracted using ethyl acetate $(15 \mathrm{~mL})$ and the extraction repeated. The ethyl acetate extracts were pooled, washed with water (twice, $30 \mathrm{~mL}$ each time) dried over anhydrous $\mathrm{Na}_{2} \mathrm{SO}_{4}$ and evaporated in vacuo.

Yield: $37.1 \%, \mathrm{mp} 170-2{ }^{\circ} \mathrm{C}, \mathrm{R}_{\mathrm{f}} 0.72$ (ethyl acetate), IR ( $\mathrm{KBr} /$ $\mathrm{cm}^{-1}$ ): 1052, 1285, 1720, 3189, 3375; ${ }^{1} \mathrm{H}-\mathrm{NMR}$ (DMSO-d $/ \mathrm{ppm}$ ): 8.78 (s, 1H, N-H), 7.75 (s, 1H, N-H), 7.32 (s, 2H, NH $), 1.45$ (m, $9 \mathrm{H},\left(\mathrm{CH}_{3}\right)_{3}$

1-(tert-butyloxycarbonylamino)-2-(4-oxo-4,5-dihydrothiazol2-yl)hydrazide (3): A mixture of compound 2 ( $1.9 \mathrm{~g}, 10 \mathrm{mmol}), \alpha$ chloroacetic acid $(0.9 \mathrm{~g}, 10 \mathrm{mmol})$ and sodium acetate $(0.8 \mathrm{~g}, 10$ $\mathrm{mmol}$ ) in ethanol was stirred for $6 \mathrm{~h}$ at reflux temperature (TLC control). The mixture was left at $-4{ }^{\circ} \mathrm{C}$ for $72 \mathrm{~h}$. Then, $n$-hexane was subsequently added and the solid was filtered. Ethyl acetate was added and the organic phase was washed with water, dried over (anhydrous sodium sulfate) and evaporated.

Yield: $78 \%, \mathrm{mp} 165-168^{\circ} \mathrm{C}, \mathrm{R}_{\mathrm{f}} 0.68$ (7:3, ethyl acetate/hexane), IR $\left(\mathrm{KBr} / \mathrm{cm}^{-1}\right)$ : 1250, 1456, 1717, 3135; ${ }^{1} \mathrm{H}-\mathrm{NMR}$ (DMSO-d $/ \mathrm{ppm}$ ): 9.39 (s, 1H, N-H), $11.30(\mathrm{~s}, 1 \mathrm{H}, \mathrm{N}-\mathrm{H}), 3.93\left(2 \mathrm{H}, \mathrm{CH}_{2}\right), 1.47$ (m, $9 \mathrm{H},\left(\mathrm{CH}_{3}\right)_{3}$.

2,N[2'(5'-dihydro-1'3-thiazol-4'-one)]hydrazide trifluoroacetate (4): to solid $(2.3 \mathrm{~g}, 10 \mathrm{mmol})$, compound $\mathbf{3}, 5 \mathrm{~mL}$ of a mixture of trifluoroacetic acid and dichloromethane (1:1) was added 
and stirred at room temperature for $1 \mathrm{~h}$. The solvent was evaporated in vacuo to produce an amorphous white residue that was collected and crystallised from ether.

Yield: $98 \%, \mathrm{R}_{\mathrm{f}} 0.65$ (ethyl acetate), IR $\left(\mathrm{KBr} / \mathrm{cm}^{-1}\right)$ : 1205, 1400, 1705, 3139; ${ }^{1} \mathrm{H}-\mathrm{NMR}$ (DMSO-d 6 ppm): 11.8 (s, 1H, N-H), 3.86 $\left(2 \mathrm{H}, \mathrm{CH}_{2}\right)$.

Synthesis of 4-thiazolidone derivatives containing an amino acid moiety - general procedure: $1.34 \mathrm{~g}(6.8 \mathrm{mmol})$ of dicyclohexylcarbodiimide, $1.0 \mathrm{~g}(5.1 \mathrm{mmol})$ of hydroxybenzotriazole and $0.13 \mathrm{~g}$ ( $1.0 \mathrm{mmol})$ of intermediate 4 were added to a stirred solution of $2.5 \mathrm{mmols}$ of the $\mathrm{N}$-protected amino acid in dimethylformamide under $0{ }^{\circ} \mathrm{C}$, and the solution was left at room temperature. The reaction mixture was filtered, and the filtrate was treated with ethyl acetate. The organic phase was washed with $\mathrm{NaHCO}_{3}$, water and aqueous $\mathrm{NaCl}$ solution, dried $\left(\mathrm{Na}_{2} \mathrm{SO}_{4}\right)$ and concentrated. The residue was treated with $n$-hexane and filtered.

2,N-[2'(5'-dihydro-1'3'-thiazole-4'-one)-yl]-1,N[2N (benzyloxycarbonylamino)ethane]hydrazide (5): Yield: 68\%, mp 152-4 ${ }^{\circ} \mathrm{C}, \mathrm{R}_{\mathrm{f}} 0.6$ (7:3, ethyl acetate/ $n$-hexane), IR $\left(\mathrm{KBr} / \mathrm{cm}^{-1}\right): 1244,1536$, 1710, 3328; ${ }^{1} \mathrm{H}-\mathrm{NMR}$ (DMSO-d $/$ ppm): 2.41 (s, 2H, CH $\left.{ }_{2}\right), 5.5$ (d, J $=7.7 \mathrm{~Hz}, 1 \mathrm{H}, \mathrm{NH}), 7.48(\mathrm{t}, J=6.2 \mathrm{~Hz}, 1 \mathrm{H}, \mathrm{NH}), 1.24-1.32(\mathrm{~s}, 9 \mathrm{H}$, $\left(\mathrm{CH}_{3}\right)_{3}, 3.66(\mathrm{~d}, J=6.2 \mathrm{~Hz}, 2 \mathrm{H}, \mathrm{CH} \alpha), 5.02\left(\mathrm{~s}, 2 \mathrm{H}, \mathrm{CH}_{2}-\mathrm{C}_{6} \mathrm{H}_{5}\right)$, 7.35-7.30 (m, 5H, Ar), $\mathrm{C}_{13} \mathrm{H}_{14} \mathrm{O}_{4} \mathrm{~N}_{4} \mathrm{~S}$.

2,N-[2'(5'-dihydro-1'3'-thiazole-4'-one)-yl]-1,N(2(S)N-[tertbutoxycarbonylamino]-2-pyrrolidine)hydrazide (6): Yield: $74 \%$, $\mathrm{mp} 105-4{ }^{\circ} \mathrm{C}, \mathrm{R}_{\mathrm{f}} 0.64$ (7:3, ethyl acetate $/ n$-hexane), $[\alpha]_{\mathrm{D}}:+1.32^{\circ}$, IR $\left(\mathrm{KBr} / \mathrm{cm}^{-1}\right): 1132,1574,1699,3328 ;{ }^{1} \mathrm{H}-\mathrm{NMR}$ (DMSO-d $\left./ \mathrm{ppm}\right): 2.81$ $\left(\mathrm{s}, 2 \mathrm{H}, \mathrm{CH}_{2}\right), 5.58(\mathrm{~d}, J=7.19 \mathrm{~Hz}, 1 \mathrm{H}, \mathrm{NH}), 1.37-1.40(\mathrm{~s}, 9 \mathrm{H}$, $\left.\left(\mathrm{CH}_{3}\right)_{3}\right), 4.57$ (dd, $\left.J=3.5 \mathrm{~Hz}, 3.8 \mathrm{~Hz}, 1 \mathrm{H} \mathrm{CH \alpha}\right), 1.83-1.91(\mathrm{~m}, 2 \mathrm{H}$, $\mathrm{CH} \beta), 2.02-2.07(\mathrm{~m}, 2 \mathrm{H}, \mathrm{CH}), 2.50(\mathrm{t}, J=3.2 \mathrm{~Hz}, 2 \mathrm{H}, \mathrm{CH})$, $\mathrm{C}_{13} \mathrm{H}_{20} \mathrm{O}_{4} \mathrm{~N}_{4} \mathrm{~S}$.

2,N-[2'(5'-dihydro-1'3'-thiazole-4'-one)-yl]-1,N(2(S)N-[tertbutoxycarbonylamino]-3-(benzyloxycarbonyl)propane) hydrazide (7): Yield: $46 \%, \mathrm{mp} 138-9{ }^{\circ} \mathrm{C}, \mathrm{R}_{\mathrm{f}} 0.65$ (7:3, ethyl acetate/ $n$-hexane), $[\alpha]_{\mathrm{D}}:+19.8^{\circ}, \mathrm{IR}\left(\mathrm{KBr} / \mathrm{cm}^{-1}\right): 1225,1501,1717,3323$; ${ }^{1} \mathrm{H}-\mathrm{NMR}$ (DMSO-d $6 / \mathrm{ppm}$ ): $2.58\left(\mathrm{~s}, 2 \mathrm{H}, \mathrm{CH}_{2}\right), 7.50(\mathrm{~d}, J=8.0 \mathrm{~Hz}$, $1 \mathrm{H}, \mathrm{NH}), 7.17$ (d, $J=8.0 \mathrm{~Hz}, 1 \mathrm{H}, \mathrm{NH}), 1.35-1.39\left(\mathrm{~s}, 9 \mathrm{H},\left(\mathrm{CH}_{3}\right)_{3}\right)$, $4.49(\mathrm{dd}, J=8.0 \mathrm{~Hz}, 8.39 \mathrm{~Hz}, 1 \mathrm{H}, \mathrm{CH} \alpha), 2.50(\mathrm{t}, J=8.3 \mathrm{~Hz}, 2 \mathrm{H}$, $\mathrm{CH}), 5.13$ (s, 2H, CH), 7.35-7.31 (m, 5H), $\mathrm{C}_{19} \mathrm{H}_{24} \mathrm{O}_{6} \mathrm{~N}_{4} \mathrm{~S}$.

2,N-[2'(5'-dihydro-1'3'-thiazole-4'-one)-yl]-1,N(2(S)N-[tertbutoxycarbonylamino]-p-[2,3-di-chloro-(benzyloxy)phenyl-3propane]hydrazide (8): Yield: $80 \%$, mp $145-147{ }^{\circ} \mathrm{C}, \mathrm{R}_{\mathrm{f}} 0.77$ (7:3, ethyl acetate $/ n$-hexane), $[\alpha]_{\mathrm{D}}:+17.6^{\circ}, \mathrm{IR}\left(\mathrm{KBr} / \mathrm{cm}^{-1}\right): 1231,1512$, 1736, 3329; ${ }^{1} \mathrm{H}-\mathrm{NMR}$ (DMSO-d $/ \mathrm{ppm}$ ): 2.83 (s, 2H, $\mathrm{CH}_{2}$ ), 5.59 (d, $J=7.7 \mathrm{~Hz}, 1 \mathrm{H}, \mathrm{NH}), 7.95(\mathrm{~s}, 1 \mathrm{H}, \mathrm{NH}), 1.23-1.32\left(\mathrm{~s}, 9 \mathrm{H},\left(\mathrm{CH}_{3}\right)_{3}\right)$, 4.48-4.56 (1H, CH $\alpha), 2.50(\mathrm{t}, J=8.7 \mathrm{~Hz}, 2 \mathrm{H}, \mathrm{CH} \beta), 5.19$ (s, $2 \mathrm{H}$, $\mathrm{CH})$, 7.44-7.49 (m, 3H, Ar), $\mathrm{C}_{24} \mathrm{H}_{26} \mathrm{O}_{5} \mathrm{~N}_{4} \mathrm{SCl}_{2}$.

2,N-[2'(5'-dihydro-1'3'-thiazole-4'-one)-yl]-1,N(2(S)N-[9. fluorenylmethoxycarbonyl]-6,N(tert-butoxycarbonylamino)hexane-hydrazide (9): Yield: $82 \%, \mathrm{mp} 117-120{ }^{\circ} \mathrm{C}, \mathrm{R}_{\mathrm{f}} 0.66$ (7:3, ethyl acetate $/ n$-hexane), $[\alpha]_{\mathrm{D}}:-22^{\circ}, \mathrm{IR}\left(\mathrm{KBr} / \mathrm{cm}^{-1}\right): 1245,1533,1626$, 3328; ${ }^{1} \mathrm{H}-\mathrm{NMR}$ (DMSO- $\left.d_{6} / \mathrm{ppm}\right): 2.81$ (s, $\left.2 \mathrm{H}, \mathrm{CH}_{2}\right), 4.38$ (d, $J=$ $7.7 \mathrm{~Hz}, 1 \mathrm{H}, \mathrm{NH}), 8.04$ (d, $\left.J=7.7 \mathrm{~Hz}, 1 \mathrm{H}, \mathrm{NH}_{2}\right), 6.71(\mathrm{~s}, 1 \mathrm{H}, \mathrm{NH})$, 1.37-1.40 (s, 9H, $\left.\left(\mathrm{CH}_{3}\right)_{3}\right), 7.37(\mathrm{~d}, \mathrm{t}, J=7.4 \mathrm{~Hz}, 7.19 \mathrm{~Hz}, 4 \mathrm{H}), 7.88$ (d, $J=7.4 \mathrm{~Hz}, 2 \mathrm{H}), 7.70(\mathrm{~m}, 2 \mathrm{H}), 4.35-4.40(\mathrm{~m}, 1 \mathrm{H}, \mathrm{CH}), 3.17-3.20$ (m, 2H), 1.27-1.29 (m, 2H), 2.49-2.51 (m, 2H), 2.89-2.91 (m, 2H), $5.56(\mathrm{~d}, J=8.3 \mathrm{~Hz}, 1 \mathrm{H}), 1.37-1.40(\mathrm{~m}, 9 \mathrm{H}), \mathrm{C}_{29} \mathrm{H}_{35} \mathrm{O}_{6} \mathrm{~N}_{5} \mathrm{~S}$.

\section{RESULTS AND DISCUSSION}

In the course of an ongoing research aiming to design aminoacylthiazolidone, we had planned an initial strategy involving condensation of thiosemicarbazide with protected amino acid residues followed by a cyclization reaction as illustrated in Scheme 1 . We used the classical carbonyl activating group-diciclohexylcarbodiimide, $\mathrm{N}$-hydroxysuccinimide, and BOP Castro's reagent (benzotriazolyloxy-tris-(dimethylamino) phosphonium hexafluorophosphate $)^{25}$ - to obtain intermediate I. Compound III was obtained after cyclization of intermediate I with chloroacetic acid in the presence of sodium acetate and ethanol as a solvent under reflux, but with low purity and yield. An efficient nucleophylic attachment of $\mathrm{NH}_{2}$-hydrazine of thiosemicarbazide to the carbonyl moiety is required. When 2-methyl-3-thiosemicarbazide was used to the condensation reaction with protected amino acids, a better result was achieved. Compounds II and IV were obtained with moderate purity and yield, but not enough to obtain a new series ${ }^{26}$.

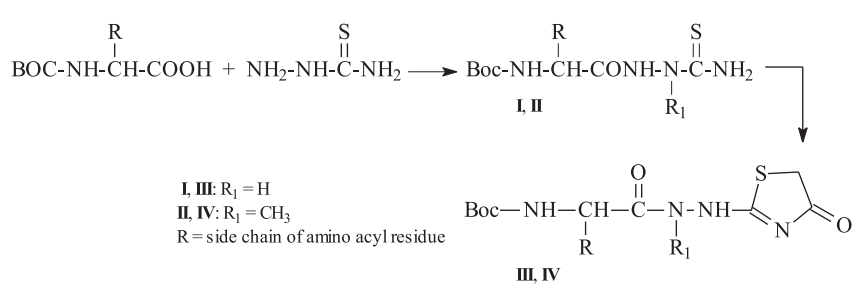

Scheme 1. Synthesis of peptidyl 4-thiazolidones

Finally, we decided to develop another optimal strategy. The preparation of new amino acyl-4-thiazolidones was achieved as illustrated in Scheme 2. The primary amine of thiosemicarbazide was protected by the Boc (tert-butyloxycarbonyl) group using the symmetrical anhydride Di-tert-butyl pyrocarbonate (Boc) ${ }_{2} \mathrm{O}$. A cyclization reaction of Boc-thiosemicarbazide was carried out under the above-mentioned conditions to obtain Boc-4-thiazolidone with $78 \%$ yield. To remove the Boc group of the heterocyclic ring, classical cleavage conditions of peptide synthesis using TFA/ $\mathrm{CH}_{2} \mathrm{Cl}_{2}(1: 1)^{27,28}$ produces unprotected 4-thiazolidone (98\% yield). The last step involves a condensation reaction between several $\alpha$-amino acids and

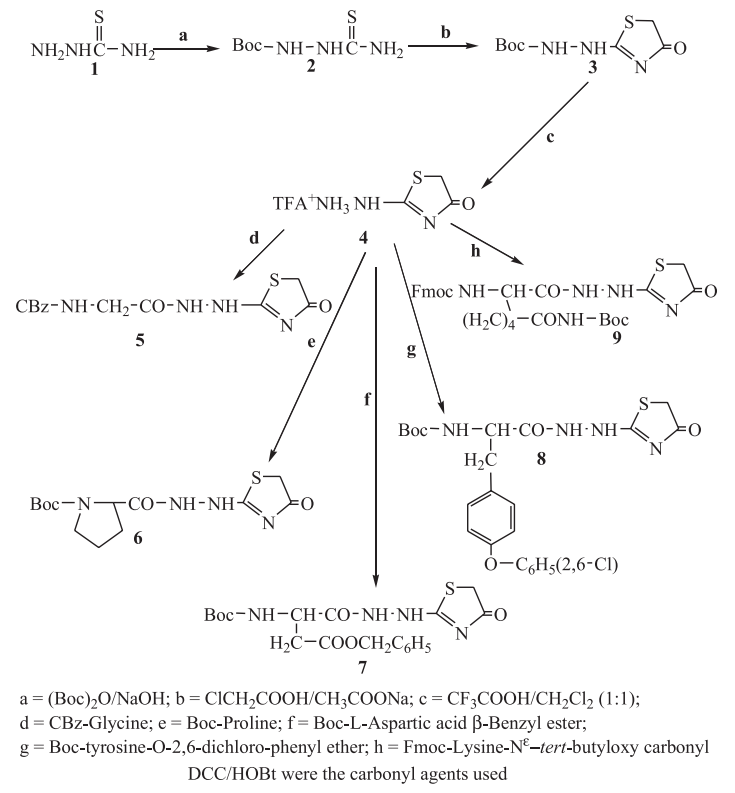

Scheme 2. Synthesis of peptidyl derivatives of 4-thiazolidones 
the amine moiety of 4-thiazolidone. DCC (dicyclohexylcarbodiimide) and HOBt (hydroxybenzotriazole) were used as carbonyl activating agents ${ }^{26-28}$.

As part of our strategy, we used amino acids with Boc (tertbutyloxycarbonyl), 9-fluorenylmethyloxycarbonyl (Fmoc) or benzyloxycarbonyl $(\mathrm{CBz})$ on the N-terminal protecting group. Lateral chains of aspartic acid were protected using a benzyl ester, lysine using a tert-butyl ester, and tyrosine using a 2,6-dichlorobenzyl ether.

The IV spectra of peptidyl derivatives exhibited $\mathrm{NH}$ and $\mathrm{C}=\mathrm{O}$ bands in the 3329-3323 and 1736-1626 $\mathrm{cm}^{-1}$ regions attributed to common CONH-N. The characteristic common lactam $\mathrm{C}=\mathrm{O}$ stretchings were observed in the $1730-1710 \mathrm{~cm}^{-1}$ region. The $\mathrm{C}=\mathrm{N}$ band appeared at 1501-1574.

The ${ }^{1} \mathrm{H}-\mathrm{NMR}$ spectra of compounds 5-9 displayed a single $\mathrm{NH}$ resonance $\left(\delta\right.$ 7.9-8.0 ppm), and the diagnostic $\mathrm{S}_{-} \mathrm{CH}_{2}(\delta 3.48-3.27$ $\mathrm{ppm}$ ). As for the amino acid moiety, all compounds showed typical signs of $\mathrm{NH}$ and the proton linked to the chiral carbon (except in the case of the proline derivative). For the NH function, a characteristic doublet due to coupling with proton of chiral carbon $(J=7.0 \mathrm{~Hz})$ appeared at $\delta 6.71-7.5 \mathrm{ppm}$. Different chemical shifts for the groups next to the chiral center were observed, especially when bulky substituents were produced.

The compounds synthesized in the present work represent a new class of aminoacyl derivatives from 4-thiazolidones scaffolds. The reactions to prepare the new series having an aminoacyl moiety are versatile and can be performed using most natural amino acids as a starting point. This suggests that this method can be used to obtain peptidyl fragments of 4-thiazolidones. The new compounds have shown promising preliminary results in terms of antitumour and antichagasic properties ${ }^{29,30}$.

\section{ACKNOWLEDGEMENTS}

We would like to express our gratitude to the Brazilian National Research Council (CNPq), the "Fundação de Amparo a Ciência do Estado de Pernambuco" (FACEPE) and Banco do Nordeste for financial support and to the Departamento de Química Fundamental, Universidade Federal de Pernambuco (UFPE), for the recording the ${ }^{1} \mathrm{H}-\mathrm{NMR}$ and IR spectra of all compounds. The authors also extend their thanks to C. R. M. Nascimento for providing technical support (CNPq \#500361/2004-5)

\section{REFERENCES}

1. Souza, M. V. N.; Ferreira, B. S.; Mendonça, J. S.; Costa, M.; Rebello, F. R.; Quim. Nova 2005, 28, 77.
2. Holla, B. S.; Malini, K. V.; Rao, B. S.; Sarojini, B. K.; Kumari, N. S.; Eur. J. Med. Chem. 2003, 38, 313.

3. Oh, C. H.; Cho, H. W.; Baek, D.; Cho, J. H.; Eur. J. Med. Chem. 2002, 37, 743

4. Kavitha, C. V.; Basappa; Swamy, S. N.; Mantelingu, K.; Doreswamy, S.; Sridhar, M. A.; Prasad, J. S.; Rangappa, K. S.; Bioorg. Med. Chem. 2006, 14, 2290.

5. Tapia, R. A.; Alegria, L.; Pessoa, C. D.; Salas, C.; Cortés, M. J.; Valderrama, J. A.; Sarciron, M. E.; Pautet, F.; Walchshofer, N.; Fillion, H.; Bioorg. Med. Chem. 2003, 11, 2175 .

6. Alves, A. J.; Leite, A. C. L.; Santana, D. P.; Beltrão, M. T.; Coelho, M. R.; Gayral, P.; Il Farmaco 1993, 48, 1167.

7. Singh, I. P.; Saxena, A.; Shanker, K.; Eur. J. Chim. Ther. 1985, 20, 283.

8. Medime, E.; Çapan, G.; Il Farmaco 1994, 49, 449.

9. Andreani, A.; Rambaldi, M.; Bonazzi, D.; Lelli, G.; Eur. J. Med. Chem. Ther. 1984, 3, 219.

10. Bordi, F.; Catellani, P. L.; Morinha, G.; Plazzi, P. V.; Silva, C.; Barocelli, E., Chiavarini, M.; Il Farmaco 1989, 44, 795.

11. Vicini, P.; Geronikaki, A.; Incerti, M.; Busonera, B.; Poni, G.; Cabras, C. A.; La Colla, P.; Bioorg. Med. Chem. 2003, 11, 4785.

12. Wipf, P.; Chem. Rev. 1995, 95, 2115.

13. Pattenden, G.; J. Heterocycl. Chem. 1992, 29, 607.

14. Boden, D. C.; Pattenden, G.; Ye, T.; Synlett 1995, 417.

15. Koeber-Plé, K.; Massiot, G.; J. Heterocycl. Chem. 1995, 32, 1309.

16. Clough, J.; Chen, S.; Gordon, E. M.; Hackbarth, C.; Lam, S.; Trias, J.; White, R. J.; Candiani, G.; Donadio, S.; Romano, G.; Ciabatti, R.; Jacobs, J. W.; Bioor. Med. Chem. Lett. 2003, 13, 3409.

17. Leite, A. C. L.; Silva, K. P.; Souza, I. A.; Araújo, J. M.; Brondani, D. J.; Eur. J. Med. Chem. 2004, 39, 1059.

18. Trouet, A.; Passioukov, A.; Derpoorten, K.; Fernandez, A. M.; AbarcaQuinones, J.; Baurain, R.; Lobl, T. J.; Oliyai, C.; Shochat, D.; Dubois, V.; Cancer Res. 2001, 61, 2843.

19. Chakravarty, P. K.; Carl, P. L. ; Weber, M. J.; Katzenellenbogen, J. A.; J. Med. Chem. 1983, 26, 633.

20. Sangapandu, S. V.; Sachdeva, S.; Jain, M.; Singh, S.; Singh, P. P.; Kaul, C. L.; Jain, R.; Bioorg. Med. Chem. 2004, 12, 239-247.

21. Aggarwal, S. K.; Gogu, S. R. S.; Rangan, R. S.; Agrawal, K.; J. Med. Chem. 1990, 33, 1505.

22. Philip, A.; Kepler, J.; Johnson, B.; Carrol, F.; J. Chem. Soc. 1988, 31, 870.

23. Arap, W.; Pasqualini, R.; Ruoslahti, E.; Science 1998, 279, 377.

24. Leite, A. C. L.; Vieira, R. F. F.; Moreira, D. R. M.; Brondani, D. J.; Srivastava, R. M.; Silva, V. F.; Junior, M. A. de M.; Mutation Res. 2005 , $588,166$.

25. Leite, A. C. L.; Santos, L. M. F.; Nascimento, D. D. S.; Sena, K. X. F. R.; Brondani, D. J.; Acta Farm. Bonaerense, 2004, 23, 117.

26. Jones, J.; The Chemical Synthesis of Peptides, Oxford University Press: New York, 1994, p. 229.

27. Leite, A. C.; Santos, L. M. F.; Anjos, J. V.; Farias, M. P. A.; Souza, I. A.; Brondani, D. J.; Heterocycl. Commun. 2003, 9, 73.

28. Machado, A.; Liria, C. W.; Proti, P. B.; Remuzgo, C.; Miranda, T. M.; Quim. Nova 2004, 27, 781.

29. Leite, A. C. L.; Santos, L. M. F.; Barbosa, F. F.; Cardoso, M. V. O.; Moreira, D. R. M.; Souza, I. A.; Biomedicine \& Pharmacother, 2006, 60, 121.

30. Leite, A. C. L.; Santos, L. M. F.; Lima, R. S.; Cardoso, M. V. O.; Moreira, D. R. M.; Brito, A. C. G.; Hernandes, M. Z.; Kiperstok, A. C.; Lima, R. S.; Soares, M. B. P.; Bioorg. Med. Chem. 2006, 14, 3749. 